\title{
Mycorrhizal fungi influence global plant biogeography
}

\author{
Camille S. Delavaux ${ }^{1,2 \star}{ }^{1}$, Patrick Weigelt ${ }^{3}$, Wayne Dawson ${ }^{4}$, Jessica Duchicela ${ }^{5}$, FranzEss ${ }^{6}$, \\ Mark van Kleunen ${ }^{7,8}$, Christian König ${ }^{1}{ }^{3}$, Jan Pergl ${ }^{9}$, Petr Pyšek ${ }^{9,10,11}$, Anke Stein ${ }^{7}$, Marten Winter ${ }^{10}{ }^{11}$, \\ Peggy Schultz ${ }^{1}$, Holger Kreft ${ }^{3}{ }^{3,12}$ and James D. Bever ${ }^{1,2}$
}

\begin{abstract}
Island biogeography has traditionally focused primarily on abiotic drivers of colonization, extinction and speciation. However, establishment on islands could also be limited by biotic drivers, such as the absence of symbionts. Most plants, for example, form symbioses with mycorrhizal fungi, whose limited dispersal to islands could act as a colonization filter for plants. We tested this hypothesis using global-scale analyses of $\sim 1.4$ million plant occurrences, including $\sim 200,000$ plant species across $\sim 1,100$ regions. We find evidence for a mycorrhizal filter (that is, the filtering out of mycorrhizal plants on islands), with mycorrhizal associations less common among native island plants than native mainland plants. Furthermore, the proportion of native mycorrhizal plants in island floras decreased with isolation, possibly as a consequence of a decline in symbiont establishment. We also show that mycorrhizal plants contribute disproportionately to the classic latitudinal gradient of plant species diversity, with the proportion of mycorrhizal plants being highest near the equator and decreasing towards the poles. Anthropogenic pressure and land use alter these plant biogeographical patterns. Naturalized floras show a greater proportion of mycorrhizal plant species on islands than in mainland regions, as expected from the anthropogenic co-introduction of plants with their symbionts to islands and anthropogenic disturbance of symbionts in mainland regions. We identify the mycorrhizal association as an overlooked driver of global plant biogeographical patterns with implications for contemporary island biogeography and our understanding of plant invasions.
\end{abstract} \footnotetext{
results from the balance of immigration, which decreases with isolation (that is, distance from the mainland), extinction, which decreases with island size $^{1}$, and speciation, which increases with island $\operatorname{size}^{2,3}$. Subsequent work has identified that environmental heterogeneity and geological and climatic history also have important effects on the diversities of island biotas ${ }^{4-7}$. Individual case studies show that biotic interactions can also influence species colonization and extinction probabilities on islands, but the generalizability of these effects is uncertain ${ }^{8,9}$. The order of arrival, resulting in priority effects ${ }^{10}$, is likely to be particularly important for mutualistic symbioses. The mycorrhizal symbiosis formed between soil fungi and most plant species is a prime candidate for priority effects because plant species vary in their dependence on the association, while mycorrhizal fungi are unlikely to establish first because they are obligately dependent on their hosts ${ }^{11,12}$.

Mycorrhizal fungi are mainly known for their role in nutrient acquisition, but also provide additional benefits for their associated plants, including pathogen resistance and soil aggregation ${ }^{13}$. Associating with mycorrhizal fungi is the ancestral state of plants, but this trait has been lost repeatedly over evolutionary history $y^{14,15}$. There are several ecological contexts in which the independence of mycorrhizal symbioses confers a competitive advantage to plants ${ }^{15,16}$. One is when mycorrhizal fungal presence is unreliable,
}

lassical island biogeography recognizes that species richnes

such as in newly formed habitats ${ }^{17,18}$. With their obligate plant host dependence, mycorrhizal fungi are not likely to establish on islands-particularly isolated oceanic islands-before their host plants. Therefore, the absence of these fungi may act as a biotic habitat filter leading to disproportionate colonization by plant species that do not rely on mycorrhizal fungi. A second context where the independence of the symbioses may confer an advantage to plants is when mycorrhizal fungi cannot grow due to environmental constraints, including anoxic soils and extreme cold ${ }^{19-22}$. The mycorrhizal plant limitation of these environmental constraints suggests that there should be fewer mycorrhizal plant species at high latitudes and altitudes. A third context that may lead to independence of mycorrhizal fungi is when the costs of the symbioses outweigh the benefits due to particularly high or low soil fertility ${ }^{23-26}$. These environmental forces may be more important where dispersal limitation is relatively unimportant, such as in mainland regions. Although a recent analysis of the distribution of arbuscular mycorrhizal fungi suggested that these fungi are not likely to be limited by dispersal ${ }^{27}$, this study did not consider islands on which dispersal limitation would be strongest. Moreover, limited dispersal of mycorrhizal fungi is supported by distribution patterns of ectomycorrhizal fungi ${ }^{28,29}$, and the performance of both arbuscular and ectomycorrhizal plants can be limited by the absence of appropriate symbionts $\mathrm{s}^{30,31}$.

${ }^{1}$ Department of Ecology and Evolutionary Biology, University of Kansas, Lawrence, KS, USA. ${ }^{2}$ Kansas Biological Survey, University of Kansas, Lawrence, KS, USA. ${ }^{3}$ Department of Biodiversity, Macroecology and Biogeography, University of Goettingen, Göttingen, Germany. ${ }^{4}$ Department of Biosciences, University of Durham, Durham, UK. ${ }^{5}$ Departamento de Ciencias de la Vida, Universidad de las Fuerzas Armadas ESPE, Sangolquí, Ecuador. ${ }^{6}$ Division of Conservation Biology, Vegetation and Landscape Ecology, University of Vienna, Vienna, Austria. 'Department of Biology, University of Konstanz, Konstanz, Germany. ${ }^{8}$ Zhejiang Provincial Key Laboratory of Plant Evolutionary Ecology and Conservation, Taizhou University, Taizhou, China. ${ }^{9}$ Institute of Botany, Department of Invasion Ecology, Czech Academy of Sciences, Průhonice, Czech Republic. ${ }^{10}$ Department of Ecology, Faculty of Science, Charles University, Prague, Czech Republic. "German Centre for Integrative Biodiversity Research (iDiv) Halle-Jena-Leipzig, Leipzig, Germany. ${ }^{12}$ Centre of Biodiversity and Sustainable Land Use, University of Goettingen, Göttingen, Germany. *e-mail: camille.delavaux@ku.edu 


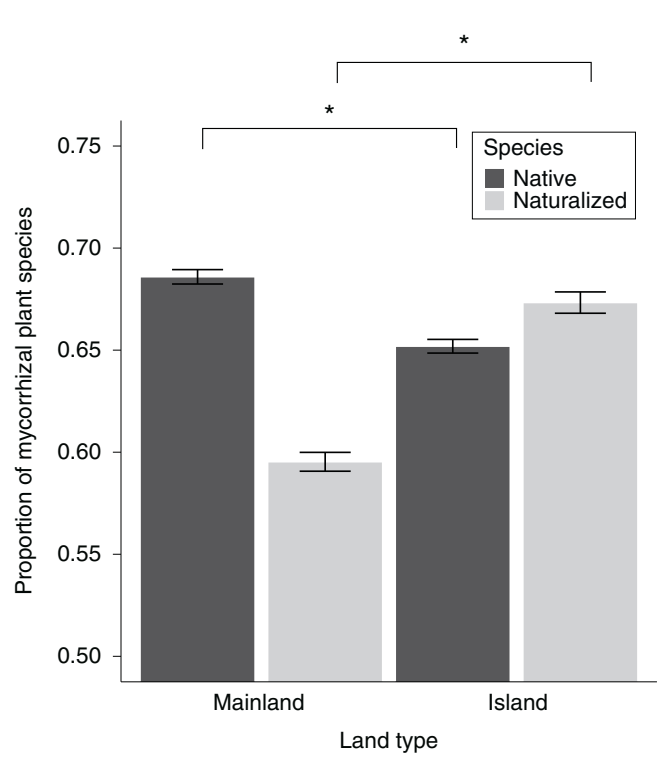

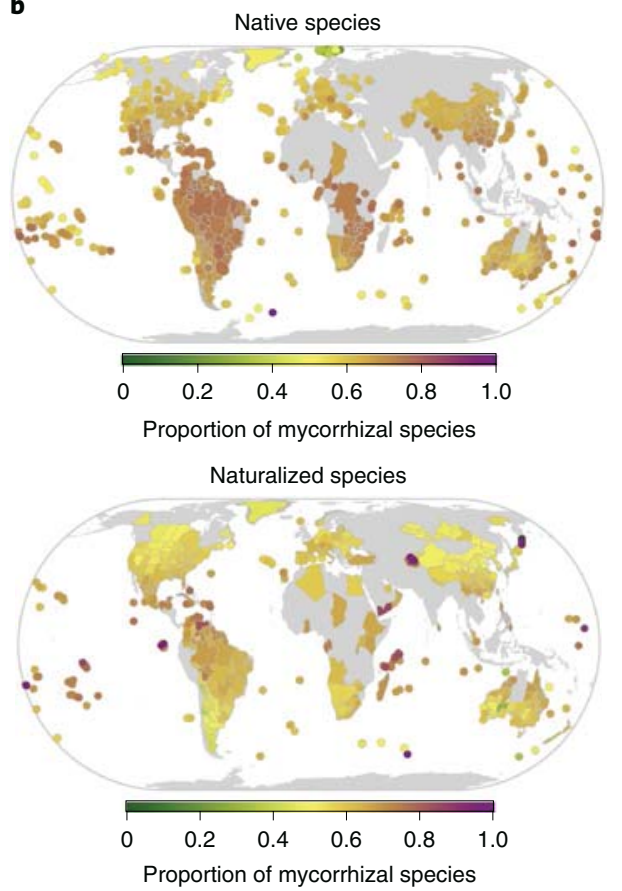

Fig. 1 | Proportion of mycorrhizal plant species in native and naturalized floras of island and mainland regions. a, For native floras (black bars), the proportions of species that are mycorrhizal are significantly greater for mainland than island regions (marginal $\left.R^{2}=0.57 ; P<0.001 ; \mathrm{GLMM}\right)$. For naturalized floras (grey bars), the proportions of species that are mycorrhizal are significantly greater for island than mainland regions (marginal $R^{2}=0.11$; $P<0.001$; native mainland, $n=1,030$; native island, $n=930$; naturalized mainland, $n=809$; naturalized island, $n=361 ; G L M M$ ). Bar heights and error bars represent means \pm s.e. Lines (labelled with asterisks) above the graph show significantly different proportions. $\mathbf{b}$, Maps of geographical regions showing the proportion of mycorrhizal plant species for native and naturalized floras included in this study.

These expected patterns of proportion of mycorrhizal species for native plant species may differ for anthropogenically driven introductions of plant species ${ }^{14,15}$. On islands, plant introductions by humans may overcome the mycorrhizal filter, as agricultural or ornamental perennial plants are commonly brought with soil and associated microbes ${ }^{32}$. This may result in naturalized floras with a greater reliance on mycorrhizal fungi ${ }^{18,33}$. Human disturbance of soils through land use may disrupt mycorrhizal fungal communities (unreliable presence) $^{34}$, thereby reducing the proportion of plants in a naturalized flora that rely on mycorrhizal fungi. This driver may be dominant in mainland regions, where soil disturbance has been shown to reduce the presence of mycorrhizal fungi ${ }^{35}$. While individual studies have found differences in the proportion of mycorrhizal plant species between native and naturalized floras ${ }^{27,35-38}$, they were mostly conducted on small scales and within mainlands. A comprehensive global analysis of factors influencing the distribution of mycorrhizal plants is required to test for general patterns.

Here, we use a global dataset of 213,710 angiosperm plant species, including $1,437,761$ plant occurrences across 1,103 regions, to test for patterns of plant specie's mycorrhizal status in island and mainland floras, for both native and naturalized plant species. To test whether a mycorrhizal filter affects island colonization, we contrast the mycorrhizal status (assigned to species in each family from each region in the same proportion as the averaged reported proportion of mycorrhizal and non-mycorrhizal species for that family) of native and naturalized species in island and mainland floras, and assess the effects of island geology, age and distance from the mainland on the proportion of species that are mycorrhizal.

\section{Results and discussion}

Our results show that mycorrhizal fungi influence global plant distributions and are associated with classic biogeographical patterns such as the latitudinal diversity gradient ${ }^{39}$ and speciesisolation relationship ${ }^{40}$. We find compelling evidence that initial colonization of islands by plants is influenced by a mycorrhizal filter; mycorrhizal species are under-represented in contemporary native island floras compared with mainland floras (Fig. 1). Specifically, we find a significant interaction between land type and mycorrhizal status, showing that the number of native mycorrhizal plant species on islands is significantly lower than on mainlands $(P<0.0001 ; z=-7.474$; generalized linear mixed model (GLMM); Supplementary Table 1, model M1). Consistent with the operation of the mycorrhizal filter, the proportion of mycorrhizal plant species on islands declines with distance from the mainland (Fig. 2a; $P<0.01$; Supplementary Table 1, model M4; generalized linear model (GLM)). Diversification of early mycorrhizal colonists may increase the proportion of native mycorrhizal species in old oceanic archipelagos, as has been observed in the Hawaiian islands ${ }^{41}$. Nonetheless, our data show no statistically significant relationship between island age and the proportion of mycorrhizal plant species $(P=0.089$; Supplementary Table 1, model M5; GLM).

For mainland native floras, variation in the proportion of mycorrhizal plant species is primarily predicted by latitude and correlated with environmental variables. Specifically, the proportion of mycorrhizal plants increases towards the equator (Fig. $2 \mathrm{c}$,d; all $P<0.001$; GLM). This strong relationship between latitude and the proportion of mycorrhizal plant species indicates that mycorrhizal plants contribute disproportionately to the classic latitudinal diversity gradient-a pattern previously reported for European floras ${ }^{38}$. This latitudinal change in the proportion of mycorrhizal plant species may reflect the arbuscular mycorrhizal ancestral state and tropical origin of major plant clades ${ }^{15}$. Alternatively, the decreasing proportion of mycorrhizal plant species towards the poles may be explained by extreme environments limiting the plant fungal symbionts in these 

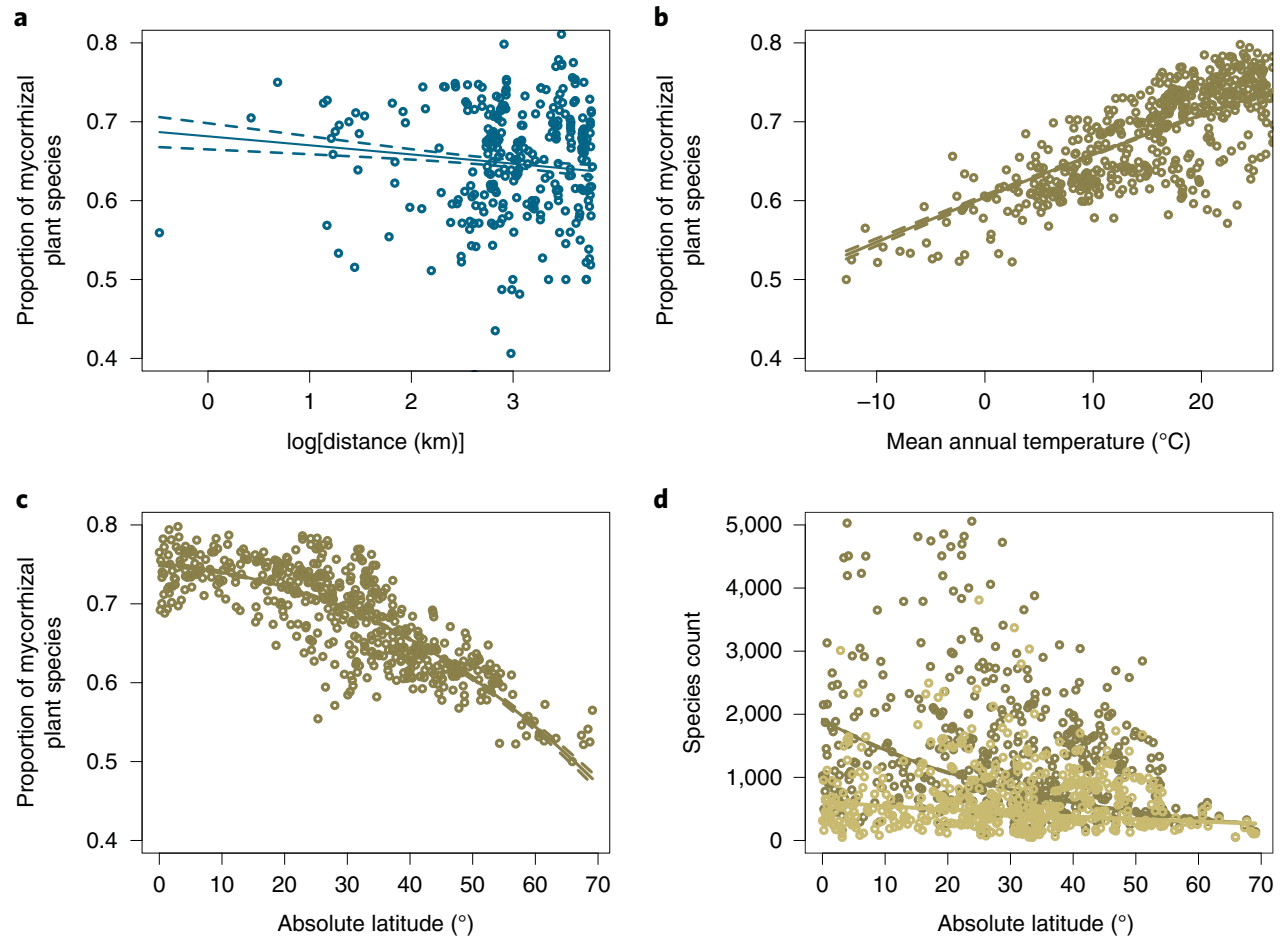

Fig. 2 | Mycorrhizal fungal associations affect biogeographical patterns of native plants. a, In island regions, increasing distance from the mainland is associated with a lower proportion of mycorrhizal plant species $(n=422 ; P<0.01 ; G L M)$. $\mathbf{b}$, In mainland regions, the proportion of mycorrhizal plant species increases with mean annual temperature $(n=515 ; P<0.001 ; G L M)$. c, In mainland regions, the proportion of mycorrhizal plant species decreases in a nonlinear manner with increasing absolute latitude ( from equator; $n=515 ; P<0.001 ; G L M$ ). $\mathbf{d}$, The relationship in c is primarily driven by mycorrhizal species (dark brown line and points) compared with non-mycorrhizal species (light brown line and points) counts ( 0 from equator; $n=515 ; P<0.001$; GLM). $\mathbf{a}$ and $\mathbf{b}$ are based on multi-predictor models including other covariables; we held other covariables at their mean to predict the variable plotted here. These model statistics can be found in Supplementary Table 1. c and $\mathbf{d}$ include only latitude and are simple pairwise models.

regions ${ }^{19}$, such as extreme cold (environmental constraints) and extensive past glacial coverage (unreliable presence). Indeed, we find that there is a significant reduction in the proportion of mycorrhizal plant species with decreasing mean annual temperature (Fig. 2b; $P<0.001$; GLM). These environmental predictors of the proportion of mycorrhizal plant species are stronger for native mainland floras than for native island floras (Supplementary Table 1, models M3 and M4, and Supplementary Figs. 2 and 3; GLM).

Naturalized island floras are disproportionately mycorrhizal compared with naturalized mainland floras (Fig. 1a; $P<0.001$; Supplementary Table 1, model M2; GLMM), suggesting anthropogenic relaxation of the mycorrhizal filter. Anthropogenic relaxation is further supported by a weakening dependence of mycorrhizal status on island isolation, as the distance from the mainland is no longer a significant predictor of species richness in naturalized island floras $(P=0.225$; Supplementary Table 1, model M7; GLM). These results are consistent with human movement of mycorrhizal fungi via transplants of mycorrhizal colonized material (for example, perennial agricultural crops, horticultural plants, sand and soil transports) to islands ${ }^{18,42}$.

In mainland regions, mycorrhizal plant species are generally under-represented in naturalized floras (Fig. 1a) compared with native floras, particularly in areas with a high proportion of native mycorrhizal plants (Fig. 3a; pseudo $R^{2}$ (coefficient of determination) $=0.286 ; P<0.0001$; GLM). This is consistent with invasions of non-mycorrhizal plant species being facilitated by the unreliable presence of mycorrhizal fungi due to large-scale anthropogenic disturbance ${ }^{35}$. Alternatively, this pattern may be a result of nutrient deposition as nutrient levels, including nitrogen and phosphorus levels, are usually higher in human-modified areas, potentially changing the cost-benefit ratio of mycorrhizal symbiosis in favour of non-mycorrhizal species. Consistent with mainland regions, we find that the proportion of mycorrhizal plants in naturalized island floras decreases with human land-use intensity $(P<0.001$; Fig. 3 b; GLM). This may be a result of islands becoming more mainlandlike, with human land use disturbing soil microbes, resulting in an unreliable presence or reduced benefit of mycorrhizal fungi. Anthropogenic influence on biogeographical patterns is also evident as environmental variables are weaker predictors for naturalized than native floras (see Supplementary Figs. 2 and 4 for all graphs of naturalized plant results; GLM).

We find consistent global patterns in the distribution of mycorrhizal plant species: mainland and island floras differ in their proportions of mycorrhizal plant species and this relationship changes with human-induced plant introductions. Furthermore, we show that in native island floras, the proportion of mycorrhizal plant species decreases with isolation (distance from the mainland). These findings are consistent with the limited dispersal of mycorrhizal fungi to islands, reducing the number of native plant species that are mycorrhizal on these islands. Finally, we find a latitudinal relationship for native mainland floras, where the proportion of mycorrhizal plant species is highest at the equator and decreases towards the poles, which is consistent with extreme cold limiting the functioning of mycorrhizal fungi ${ }^{20-22}$. We suggest that these patterns are mediated by the mycorrhizal symbiosis. Alternatively, these biogeographical patterns might be caused in part by traits that co-vary with the mycorrhizal status of plants ${ }^{43,44}$. For these co-varying traits to explain our results, non-mycorrhizal plants would need to exhibit greater dispersal ability or greater cold tolerance compared with their mycorrhizal counterparts. To date, we know of no evidence of 

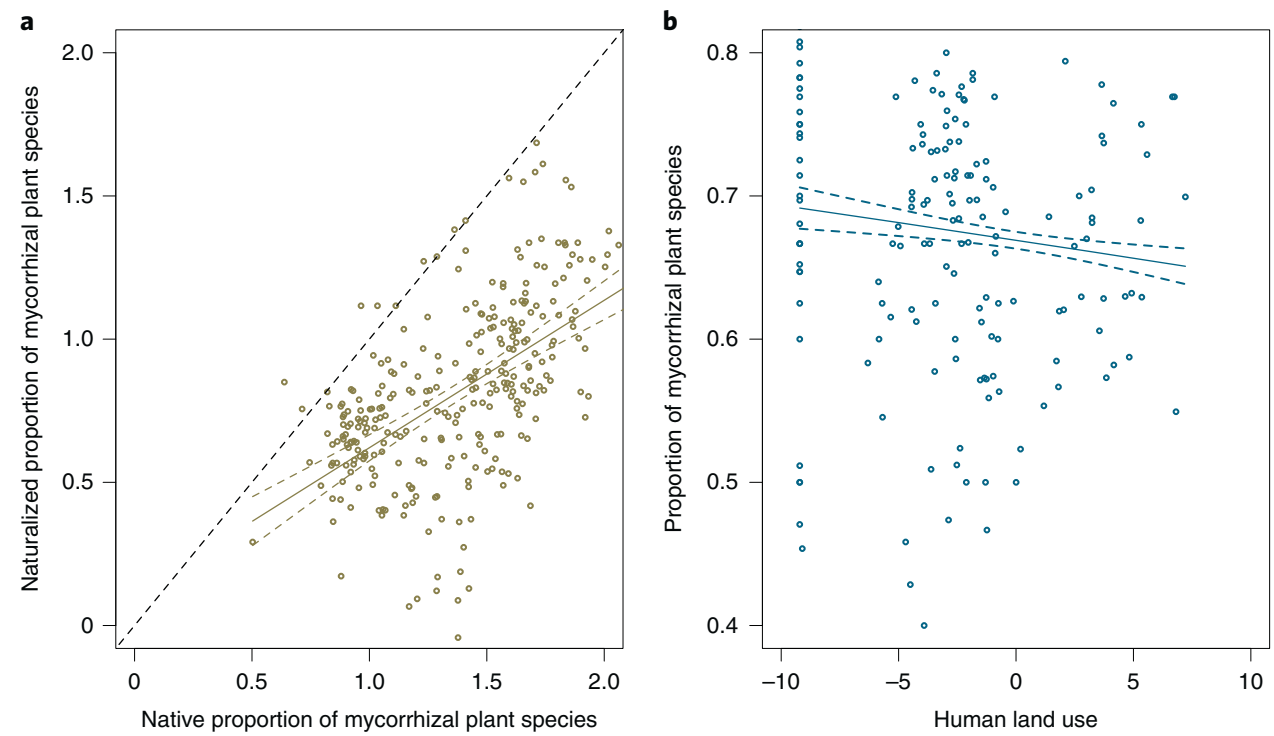

Fig. 3 | Mycorrhizal fungal associations differentially impact the biogeography of naturalized floras. a, In mainland regions, the proportion of mycorrhizal plant species in the naturalized flora is lower than in the native flora, particularly in areas with a high proportion of native mycorrhizal plants (native mainland, $n=515$; naturalized mainland, $n=294 ; P<0.001 ; G L M)$. $\mathbf{b}$, On islands, the proportion of mycorrhizal plant species decreases with human land use-a composite variable resulting from the sum of the log-transformed urban and managed land area $(n=177 ; P=0.001 ; G L M)$.

such covariance. As there is clear evidence for fungal dispersal limitation $^{29-31}$ and limits of fungal efficiency under cold environmental conditions $\mathrm{s}^{20-22}$, we contend that our results are probably mediated by mycorrhizal fungal availability and functioning.

We show that native and naturalized floras worldwide differ in their composition in conjunction with mycorrhizal association, with differing proportions in island versus mainland regions. Proportions of mycorrhizal plant species in mainland regions vary strongly along climatic and latitudinal gradients, with the proportion of mycorrhizal plant species being highest near the equator and decreasing towards the poles, suggesting that mycorrhizal species contribute disproportionately to the classical diversity-latitude relationship $^{39}$. For islands, we find that the composition of native floras reflects the mycorrhizal filter of plant colonization success; with the initial absence of their mycorrhizal symbionts, mycorrhizal plants are disproportionally filtered during island colonization. Humaninfluenced movement of naturalized species with their mycorrhizal symbionts to islands may alleviate mycorrhizal dispersal limitation, thereby weakening isolation-by-distance effects. While further work examining the mycorrhizal status of plants, particularly in the tropics, is necessary to confirm these patterns, our results suggest that mycorrhizal fungi influence global plant biogeography, including island biogeography and the species richness latitudinal gradient, and influence patterns of human-mediated plant introductions.

\section{Methods}

Plant distribution data and floristic status. Plant species occurrence data across 1,103 regions around the globe (mostly administratively defined regions, such as countries and provinces or islands), native status (native versus naturalized) and all additional parameters associated with regional characteristics were extracted from the Global Alien Naturalized Flora (GloNAF ${ }^{45}$; Supplementary Note 1) and Global Inventory of Floras and Traits $\left(\right.$ GIFT $\left.^{46}\right)$ databases. Naturalized is defined as non-native species that form self-sustaining populations in new regions ${ }^{47}$. From the GloNAF database, we included only regions for which the available species lists had a completeness level of 2 (50-90\% of naturalized species included) and 3 ( $>90 \%$ naturalized species included). From the GIFT database, we only used regions for which checklists of native angiosperms were available. When there were overlapping regions, the smaller regions were kept if they were greater than $100 \mathrm{~km}^{2}$ for mainland regions; for islands, the smaller units were always preferred. Finally, we removed islands for which the island geology (that is, volcanic, floor, shelf, fragment, and so on) was undetermined. After all cleaning of the data, we had a total of $1,437,761$ plant occurrences across 1,103 unique regions. Our final data included 133,491 plant occurrences in 574 regions from the GloNAF dataset and 1,304,270 plant occurrences in 979 regions from the GIFT dataset.

Mycorrhizal status. The mycorrhizal status of the 1,437,761 plant occurrences included in this study was determined by assigning each species to its plant family according to theplantlist.org, incorporating classification from Angiosperm Phylogeny Group IV ${ }^{48}$. We relied on family proportions of known mycorrhizal and non-mycorrhizal species to assign mycorrhizal status to species in this study. Specifically, within each region, species in a family were assigned mycorrhizal or non-mycorrhizal status in the same proportion as that family (Supplementary Table 2). Given the geographical bias in knowledge of species-level mycorrhizal status (bias towards heavily studied temperate systems), this family-level assignment was the most rigorous method we could employ. We used three review papers to determine the plant family consensus proportion mycorrhizal status $^{15,20,49}$. While concerns have been raised over incorrect classification in these review papers ${ }^{22}$, which cannot be addressed at this time due to the lack of speciesspecific corrections, potential errors are not likely to have large effects on a global database. If species in a family were arbuscular mycorrhizal, ectomycorrhizal, ericoid mycorrhizal, orchid mycorrhizal or half arbuscular mycorrhizal and half ectomycorrhizal, we classified these as 'mycorrhizal'. Different classifications and proportions between the reference papers were accounted for by using the average consensus proportions for each mycorrhizal category (mycorrhizal, non-mycorrhizal, and equally split arbuscular mycorrhizal and non-mycorrhizal (AMNM); see below) across the three references (excluding data for which values were not reported). We determined the consensus proportion of sampled species in each family that were mycorrhizal, non-mycorrhizal or ambiguous (AMNM). We ran each of our analyses twice, putting all ambiguous species (AMNM) as either mycorrhizal or non-mycorrhizal. Our initial distribution dataset was reduced to species for which we had family-level mycorrhizal data, resulting in the data described in the section 'Plant distribution data and floristic status' above. Specifically, 142,164 unique observations (unique species-location combinations) out of 2,000,000 were removed due to a lack of mycorrhizal data. Nonetheless, we find a slightly higher proportion of omitted data points at the highest latitudes these omissions are from Compositae (46) and Leguminosae (3). Full details of families and corresponding consensus proportions of mycorrhizal status can be found in Supplementary Table 2.

Explanatory variables. Explanatory variables for each of the regions were extracted from the GIFT database. For details of environmental data collection, see Weigelt et al. ${ }^{46}$. Explanatory variables include land type (mainland or island), latitude and longitude of the region's centroid, area $\left(\mathrm{km}^{2}\right)$, mean annual temperature $\left({ }^{\circ} \mathrm{C}\right)$, mean annual precipitation $(\mathrm{mm})^{50}$, maximum elevation range (that is, the difference between the lowest and highest elevation $(\mathrm{m}))^{51}$, 
human population density $\left(n \mathrm{~km}^{-2}\right)^{52}$ and two land-use metrics (cultivated and managed vegetation, and urban land-use area, combined as a sum following $\log$-transformation to form the new variable 'human land use' in our analyses) ${ }^{53}$. For islands, we also included their distance from the nearest mainland $(\mathrm{km})$ as a measure of island isolation ${ }^{40}$, geological origin (referred to in the model results as geology; oceanic or non-oceanic) and island age (millions of years; only meaningfully quantified for oceanic islands). We included non-oceanic islands in the group of oceanic islands if they were covered with ice (at least $80 \%$ ) during the Last Glacial Maximum ${ }^{53}$ because the land would have characteristics of a newly formed oceanic island after the plant and fungal communities were destroyed by glaciation.

Statistical analysis. To test broad-scale patterns of mycorrhizal-plant distributions, we modelled regional plant species richness (counts) for mycorrhizal and nonmycorrhizal plants in two analyses (separately for native and naturalized plants; Fig. 1a). For these analyses, we used GLMMs. We chose a Poisson distribution, as the response variable-species richness-is count data. The fixed effects were mycorrhizal status, land type (mainland or island) and their interaction. The random effects were region nested within land type (mainland or island) and mycorrhizal status nested within region nested within land type (mainland or island). We ran this model separately for native (model M1) and naturalized (model M2) plant species richness as response variables. The sample size $(n)$ in these two models represents a unique regional combination of native status (native or naturalized) and mycorrhizal status (mycorrhizal or non-mycorrhizal). Of the 4,492 observations in this dataset, 2,246 involved native floras and 2,246 involved naturalized floras. This corresponded to 1,123 regions with native data and 1,123 regions with naturalized data. To create Fig. 1, we converted our count estimates from the model to proportions.

To investigate drivers of mycorrhizal status of native and naturalized plants in mainland and island floras, we used the proportion of mycorrhizal species at each region as the response variable. For this analysis, we used GLMs with a logit link function, assuming a binomial distribution of the response variable. For these models, we took the natural log of area, human population density, distance from the nearest mainland, elevation range and island age to normalize the distributions. For the native mainland model (M3), we included area, mean annual precipitation, mean annual temperature and elevation range. For the native island model (M4), we included area, distance from the mainland, precipitation, temperature, elevation range and geology, as well as the interaction between distance from the mainland and geology (oceanic or non-oceanic). The tested variables were informed through previous study of these variables with this dataset ${ }^{5}$, as well as other island biogeographical studies ${ }^{54,55}$. As the presence of naturalized species is likely to be driven by human activities, the naturalized mainland model (M6) and naturalized island model (M7) included human population density in addition to the explanatory variables included in the corresponding models for native species. Finally, to account for the effect of island age, we additionally analysed the subset of oceanic islands for which we had age data. For the models of native floras including island age (M5; $n=246)$, we included area, distance from the mainland, age, precipitation, temperature and elevation range. For the models of naturalized island floras including age (M8; $n=97)$, we additionally included human population density. The results of these main models are presented in Supplementary Table 1 (M1-M8). In these and all models excluding M1 and M2, $n$ values are true numbers representing unique regions. Before any further subsetting, this dataset covered a total of 1,103 regions (same as observations); 979 regions had data for native flora and 574 regions had data for naturalized flora.

To explore linear and nonlinear latitudinal patterns in mycorrhizal distribution in more detail, we re-ran all models including only absolute latitude and absolute latitude squared. We also ran models to investigate anthropogenic drivers of mycorrhizal status in naturalized plants only. For these models, we included a combined variable of urban land-use area and cultivated and managed vegetation, termed 'human land use' (the sum of both variables). To assess the robustness of our results in the face of the uncertainty in mycorrhizal status assignment, we re-ran all models to assign ambiguous (AMNM) plants to non-mycorrhizal instead of mycorrhizal assumed in the original models. Here, we report the statistics from models in which AMNM plants were assigned to mycorrhizal (Supplementary Table 1 and Supplementary Figs. 2-4). For models where AMNM plants were assigned to non-mycorrhizal, see Supplementary Table 3 and Supplementary Figs. 5-7).

Before running all models, we removed regions where plant coverage was unreliable. We considered this to be the case when there was a zero in the total calculated mycorrhizal or non-mycorrhizal species counts in this region. The main cause of this was incomplete family coverage of mycorrhizal status; if we did not have information on the mycorrhizal status of all specie's families in a region, these regions would result in an incorrect sum of zero mycorrhizal and zero non-mycorrhizal plant species. We removed these regions because this zero value was not representative of the entire region. We also removed island regions where geology was undetermined before the analyses. We corrected for overdispersion in GLMs using a quasi-binomial or quasi-Poisson family model. In addition, most of our model residuals showed spatial autocorrelation, as tested using Moran's I, which is expected in global-scale models with spatially clustered geographical regions. We corrected for this spatial autocorrelation by creating a new variable (spatial autocovariate) that incorporates a matrix of longitude and latitude coordinates of the regions ${ }^{56}$ in the spdep package in $\mathrm{R}^{57}$. After checking for spatial autocorrelation in our corrected models, some models still showed spatial autocorrelation (as determined using Moran's I), but all spatial autocorrelation was reduced substantially (correlograms shown in Supplementary Figs. 4 and 7). All models and summary statistics were run in R 3.4.1 (ref. ${ }^{58}$ ) in the lme4 package ${ }^{59}$.

\section{References}

1. MacArthur, R. H. \& Wilson, E. The Theory of Island Biogeography (Princeton Univ. Press, Princeton, 1967).

2. Losos, J. B. \& Schluter, D. Analysis of an evolutionary species-area relationship. Nature 408, 847-850 (2000).

3. Kisel, Y. \& Barraclough, T. G. Speciation has a spatial scale that depends on levels of gene flow. Am. Nat. 175, 316-334 (2010).

4. Borregaard, M. K. et al. Oceanic island biogeography through the lens of the general dynamic model: assessment and prospect. Biol. Rev. 92, $830-853$ (2017)

5. Kreft, H., Jetz, W., Mutke, J., Kier, G. \& Barthlott, W. Global diversity of island floras from a macroecological perspective. Ecol. Lett. 11, 116-127 (2008).

6. Weigelt, P., Steinbauer, M. J., Cabral, J. S. \& Kreft, H. Late Quaternary climate change shapes island biodiversity. Nature 532, 99-102 (2016).

7. Whittaker, R. J., Triantis, K. A. \& Ladle, R. J. A general dynamic theory of oceanic island biogeography. J. Biogeogr. 35, 977-994 (2008).

8. Losos, J. B. \& Ricklefs, R. E. The theory of Island Biogeography Revisited (Princeton Univ. Press, Princeton, 2009).

9. Onstein, R. E. et al. Frugivory-related traits promote speciation of tropical palms. Nat. Ecol. Evol. 1, 1903-1911 (2017).

10. Fukami, T. Historical contingency in community assembly: integrating niches, species pools, and priority effects. Annu. Rev. Ecol. Evol. Syst. 46, 1-23 (2015).

11. Bever, J. D., Westover, K. M. \& Antonovics, J. Incorporating the soil community into plant population dynamics: the utility of the feedback approach. Ecology 85, 561-573 (1997).

12. Van der Heijden, M. G., Bardgett, R. D. \& van Straalen, N. M. The unseen majority: soil microbes as drivers of plant diversity and productivity in terrestrial ecosystems. Ecol. Lett. 11, 296-310 (2008).

13. Delavaux, C. S., Smith-Ramesh, L. M. \& Kuebbing, S. E. Beyond nutrients: a meta-analysis of the diverse effects of arbuscular mycorrhizal fungi on plants and soils.Ecology 98, 2111-2119 (2017).

14. Redecker, D., Kodner, R. \& Graham, L. E. Glomalean fungi from the Ordovician. Science 289, 1920-1921 (2000).

15. Maherali, $H$. et al. Mutualism persistence and abandonment during the evolution of the mycorrhizal symbiosis. Am. Nat. 188, E113-E125 (2016).

16. Smith, S. E. \& Read, D. J. Mycorrhizal Symbiosis (Academic Press, Oxford, UK, 2008).

17. Davison, J. et al. Hierarchical assembly rules in arbuscular mycorrhizal (AM) fungal communities. Soil Biol. Biochem. 97, 63-70 (2016).

18. Dickie, I. A. et al. The emerging science of linked plant-fungal invasions. New Phytol. 215, 1314-1332 (2017).

19. Miller, R. M., Smith, C. I., Jastrow, J. D. \& Bever, J. D. Mycorrhizal status of the genus Carex (Cyperaceae). Am. J. Bot. 86, 547-553 (1999).

20. Brundrett, M. C. Mycorrhizal associations and other means of nutrition of vascular plants: understanding the global diversity of host plants by resolving conflicting information and developing reliable means of diagnosis. Plant Soil 320, 37-77 (2009).

21. Tedersoo, L. Biogeography of Mycorrhizal Symbiosis Vol. 230 (Springer, Cham, Switzerland, 2017).

22. Brundrett, M. C. \& Tedersoo, L. Evolutionary history of mycorrhizal symbioses and global host plant diversity.New Phytol. 220, 1108-1115 (2018).

23. Lambers, H., Raven, J. A., Shaver, G. R. \& Smith, S. E. Plant nutrientacquisition strategies change with soil age. Trends Ecol. Evol. 23, 95-103 (2008). 
24. Jiang, S. et al. Dynamics of arbuscular mycorrhizal fungal community structure and functioning along a nitrogen enrichment gradient in an alpine meadow ecosystem.New Phytol. 220, 1222-1235 (2018).

25. Abbott, K. C. et al. Spatial heterogeneity in soil microbes alters outcomes of plant competition. PLoS ONE 10, e0125788 (2015).

26. Steidinger, B. S. \& Bever, J. D. The coexistence of hosts with different abilities to discriminate against cheater partners: an evolutionary game-theory approach. Am. Nat. 183, 762-770 (2014).

27. Davison, J. et al. Global assessment of arbuscular mycorrhizal fungus diversity reveals very low endemism. Science 349, 970-973 (2015).

28. Tedersoo, L. et al. Global diversity and geography of soil fungi. Science $\mathbf{3 4 6}$, 1256688 (2014).

29. Peay, K. G., Schubert, M. G., Nguyen, N. H. \& Bruns, T. D. Measuring ectomycorrhizal fungal dispersal: macroecological patterns driven by microscopic propagules. Mol. Ecol. 21, 4122-4136 (2012).

30. Peay, K. G. Timing of mutualist arrival has a greater effect on Pinus muricata seedling growth than interspecific competition. J. Ecol. 106, 514-523 (2018).

31. Koziol, L. et al. The plant microbiome and native plant restoration: the example of native mycorrhizal fungi.BioScience 68, 996-1006 (2018).

32. Richardson, D. M., Allsopp, N., D’Antonio, C. M., Milton, S. J. \& Rejmánek, M. Plant invasions-the role of mutualisms. Biol. Rev. 75, 65-93 (1999).

33. Callaway, R. M., Thelen, G. C., Rodriguez, A. \& Holben, W. E. Soil biota and exotic plant invasion. Nature 427, 731-733 (2004).

34. Oehl, F. et al. Impact of land use intensity on the species diversity of arbuscular mycorrhizal fungi in agroecosystems of Central Europe. Appl. Environ. Microbiol. 69, 2816-2824 (2003).

35. Pringle, A. et al. Mycorrhizal symbioses and plant invasions. Annu. Rev. Ecol. Evol. Syst. 40, 699-715 (2009).

36. Bunn, R. A., Ramsey, P. W. \& Lekberg, Y. Do native and invasive plants differ in their interactions with arbuscular mycorrhizal fungi? A meta-analysis. J. Ecol. 103, 1547-1556 (2015).

37. Reinhart, K. O., Lekberg, Y., Klironomos, J. \& Maherali, H. Does responsiveness to arbuscular mycorrhizal fungi depend on plant invasive status?. Ecol. Evol. 7, 6482-6492 (2017).

38. Bueno, C. G. et al. Plant mycorrhizal status, but not type, shifts with latitude and elevation in Europe. Glob. Ecol. Biogeogr. 26, 690-699 (2017).

39. Hillebrand, H. On the generality of the latitudinal diversity gradient. Am. Nat. 163, 192-211 (2004).

40. Weigelt, P. \& Kreft, H. Quantifying island isolation-insights from global patterns of insular plant species richness. Ecography 36, 417-429 (2013).

41. Koske, R., Gemma, J. \& Flynn, T. Mycorrhizae in Hawaiian angiosperms: a survey with implications for the origin of the native flora.Am. J. Bot. 79, 853-862 (1992).

42. Vellinga, E. C., Wolfe, B. E. \& Pringle, A. Global patterns of ectomycorrhizal introductions. New Phytol. 181, 960-973 (2009).

43. Cornelissen, J., Aerts, R., Cerabolini, B., Werger, M. \& Van Der Heijden, M. Carbon cycling traits of plant species are linked with mycorrhizal strategy. Oecologia 129, 611-619 (2001).

44. Powell, J. R., Riley, R. C. \& Cornwell, W. Relationships between mycorrhizal type and leaf flammability in the Australian flora. Pedobiologia 65, 43-49 (2017).

45. Van Kleunen, M. et al. Global exchange and accumulation of non-native plants. Nature 525, 100-103 (2015).

46. Weigelt, P., König, C. \& Kreft, H. GIFT- A Global Inventory of Floras and Traits; https://doi.org/10.1101/535005 (2019).

47. Van Kleunen, M. et al. Global exchange and accumulation of non-native plants.Nature 525, 100-103 (2015)

48. Byng, J. W. et al. An update of the Angiosperm Phylogeny Group classification for the orders and families of flowering plants: APG IV. Bot. J. Linn. Soc. 181, 1-20 (2016).
49. Gerdemann, J. Vesicular-arbuscular mycorrhiza and plant growth. Annu. Rev. Phytopathol. 6, 397-418 (1968).

50. Karger, D. N. et al. Climatologies at high resolution for the Earth's land surface areas. Sci. Data 4, 170122 (2017).

51. Danielson, J. J. \& Gesch, D. B. Global Multi-resolution Terrain Elevation Data 2010 (GMTED2010) Report 2331-1258 (US Geological Survey, 2011)

52. Socioeconomic Data and Applications Center (SEDAC).Gridded Population of the World, (GPW), v3; https://doi.org/10.7927/H4639MPP (Center for International Earth Science Information Network, 2015).

53. Tuanmu, M. N. \& Jetz, W. A global 1-km consensus land-cover product for biodiversity and ecosystem modelling. Glob. Ecol. Biogeogr. 23, 1031-1045 (2014)

54. Kueffer, C. et al. A global comparison of plant invasions on oceanic islands. Perspect. Plant Ecol. Evol. Syst. 12, 145-161 (2010).

55. Triantis, K. A., Economo, E. P., Guilhaumon, F. \& Ricklefs, R. E. Diversity regulation at macro-scales: species richness on oceanic archipelagos. Glob. Ecol. Biogeogr. 24, 594-605 (2015).

56. Crase, B., Liedloff, A. C. \& Wintle, B. A. A new method for dealing with residual spatial autocorrelation in species distribution models. Ecography 35, 879-888 (2012).

57. Bivand, R. \& Piras, G. Comparing Implementations of Estimation Methods for Spatial Econometrics (American Statistical Association, Alexandria, VA, USA, 2015).

58. R Core Team. R: A language and Environment for Statistical Computing (R Foundation for Statistical Computing, Vienna, Austria. 2016).

59. Bates, D., Maechler, M., Bolker, B. \& Walker, S. Fitting linear mixed-effects models using lme4. J. Stat. Softw. 67, 1-48 (2015).

\section{Acknowledgements}

We acknowledge W. Jetz, D. Sax, A. Mehring and M. Heard for introducing us to collaborators, and S. Queenborough for statistical support at the start of the project. We also acknowledge support from the US NSF (DEB-1556664 to J.D.B., P.S. and C.S.D.), National Geographical Society (WW-036ER-17 to C.S.D.), German DFG (MvK: project 264740629; MW project FZT 118), Czech Centre of Excellence Plant Diversity Analysis and Synthesis Centre (14-36079G to J.P. and P.P.), Czech Academy of Sciences (RVO 67985939 to J.P. and P.P.) and Austrian Science Foundation (I2086B16 to F.E.)

\section{Author contributions}

C.S.D. and J.D.B. designed the study. C.S.D. and J.D.B. led the statistical analysis and writing, with continued contributions from P.W. and H.K. C.S.D. collected all of the mycorrhizal data. All other authors collected the remaining plant and environmental data and edited the manuscript.

\section{Competing interests}

The authors declare no competing interests. 